\title{
Role of computed tomography imaging for transcatheter valvular repair/insertion
}

\author{
See Hooi Ewe • Robert J. Klautz • \\ Martin J. Schalij • Victoria Delgado
}

Received: 24 January 2011 / Accepted: 3 February 2011/Published online: 26 February 2011

(C) The Author(s) 2011. This article is published with open access at Springerlink.com

\begin{abstract}
During the last decade, the development of transcatheter based therapies has provided feasible therapeutic options for patients with symptomatic severe valvular heart disease who are deemed inoperable. The promising results of many nonrandomized series and recent landmark trials have increased the number of percutaneous transcatheter valve procedures in high operative risk patients. Pre-procedural imaging of the anatomy of the aortic or mitral valve and their spatial relationships is crucial to select the most appropriate device or prosthesis and to plan the percutaneous procedure. Multidetector row computed tomography provides 3-dimensional volumetric data sets allowing unlimited plane reconstructions and plays an important role in pre-procedural screening and procedural planning. This review will describe the evolving role of multidetector row computed tomography in patient selection and strategy planning of transcatheter aortic and mitral valve procedures.
\end{abstract}

Keywords Transcatheter heart valve implantation . Computed tomography $\cdot$ Imaging

S. H. Ewe · R. J. Klautz · M. J. Schalij · V. Delgado ( $\square)$ Department of Cardiology and Cardio-Thoracic Surgery, Leiden University Medical Center, Albinusdreef 2, 2333 ZA Leiden, The Netherlands e-mail: v.delgado@lumc.nl

\section{Introduction}

During the last decade, the development of transcatheter based therapies has provided feasible therapeutic options for patients with symptomatic severe valvular heart disease who are deemed inoperable. The promising results of many nonrandomized series and recent landmark trials, such as the PARTNER trial with the Edwards SAPIEN transcatheter aortic valve prosthesis (Edwards Lifesciences, Irvine, CA, USA) or the EVEREST II trial with the MitraClip device (Abbott Vascular, Structural Heart, Menlo Park, CA, USA), have increased the number of percutaneous transcatheter valve procedures in high operative risk patients $[1,2]$.

In contrast to open heart surgery where direct inspection of the valve is possible, the decision for device/prosthesis selection and planning of the percutaneous procedure is mainly based on pre-procedural imaging of the anatomy of the aortic or mitral valve and their spatial relationships. These data are usually acquired using 2-dimensional (2D) imaging modalities such as conventional echocardiography and/or invasive angiography. However, 2D echocardiography imaging relies on geometrical assumptions that may reduce the accuracy of the measurements while fluoroscopy has limited soft-tissue resolution. In contrast, advanced cardiovascular imaging modalities such as multidetector row computed tomography (MDCT), provide detailed information on the aforementioned anatomy. MDCT, which has the advantage 
of 3-dimensional (3D) volumetric data sets allowing unlimited plane reconstructions, plays an important role in pre-procedural screening and procedural planning with the aim of minimizing proceduralrelated complications. This review will describe the evolving role of MDCT in patient selection and strategy planning of transcatheter aortic and mitral valve procedures.

\section{Transcatheter aortic valve implantation}

Transcatheter aortic valve implantation (TAVI) has emerged as an effective alternative in high risk patients with symptomatic severe aortic stenosis (AS). Recently, this percutaneous intervention has demonstrated superior survival at 1 year over standard therapy (69.3\% vs. $49.3 \%$, with an absolute $20 \%$ increase with TAVI procedure) in a selected group of patients with AS who were deemed not suitable for aortic valve surgery [1]. In addition, a recent review involving more than 2,000 patients receiving TAVI reported an implantation success of $94 \%$ and a 30-day survival rate of $89 \%$ [3]. Accurate assessment of the anatomy of the peripheral arteries and aorta, together with the anatomy of the aortic valve, aortic annular and root dimensions, are the key determinants of procedural feasibility and safety.

Pre-procedural patient evaluation

The multidisciplinary pre-procedural evaluation of patients who are candidates for TAVI includes the assessment of the aortic valvular complex, including the aortic valve and aortic root (for determination of anatomical suitability and prosthesis sizing), and the anatomy of the peripheral arteries and aorta (for determination of the access site) (Table 1).

First, assessment of the aortic valve should begin with confirmation of the aortic valve morphology. Usually, this can easily be identified from the shortaxis view on transthoracic echocardiography, which remains the initial modality of choice to assess the aortic valve pathology and its hemodynamic consequences [4]. However, in patients with poor acoustic windows and/or in the presence of heavy calcification, differentiating tricuspid from bicuspid valvular anatomy may be challenging [5]. This information is important before the procedure as it is currently not recommended to perform TAVI on bicuspid valves due to the potential risk of an unfavorable deployment $[6,7]$. In a recent study of 50 patients with AS (17 bicuspid and 33 tricuspid) [8], transthoracic echocardiography was unable to identify the anatomy of the valve in 10 patients $(20 \%)$ due to extensive calcification. In contrast, MDCT was able to provide direct visualisation of the aortic valve and thus could correctly identify the valve anatomy in 49 of 50 cases (98\%) [8]. Furthermore, the accuracy of the patient selection process can be further improved with additional systolic reconstruction using ECG-gating, which permits differentiation between a bicuspid valve with raphe and a tricuspid valve [9].

Next, the assessment of the extent and location of the aortic valve calcification is important before the TAVI procedure. With high spatial resolution and the possibility of direct visualization of the aortic valve, MDCT allows detailed analysis of the quantification and localization of aortic valve calcification (Fig. 1). Several studies [10-12] have indicated the significance of aortic valve calcification and its specific location, as assessed by MDCT, in relation to the presence of post-procedural aortic regurgitation. For example, in a study of 100 patients who underwent TAVI with self-expandable devices, John et al. [11] demonstrated a strong linear correlation $(r=0.86$, $P<0.001)$ between the degree of aortic regurgitation immediately post-TAVI and the severity of calcification in the device "landing zone", defined as the area extending from the left ventricular outflow tract to the aortic valvular cusps. Similar findings were reported in a series of 53 patients undergoing TAVI [10] whereby moderate post-procedural aortic regurgitation following the implantation of balloonexpandable valves was found in patients who exhibited more calcification of the native aortic valves, especially at the valve commissures (Fig. 1). It has been suggested that bulky calcification may pose resistance during the deployment of the prosthesis, resulting in paravalvular leakage arising from the gap between the prosthesis and the native valve [13]. In addition, very bulky calcification at the edge of native valvular leaflets has been related to increased risk of coronary occlusion when it is displaced over the coronary ostium [14]. Furthermore, TAVI has to be performed with caution when there is heavy calcification in the sinotubular junction as it may cause restriction during balloon expansion at the aortic end, 
Table 1 Evaluation before transcatheter aortic valve implantation: anatomic requirements of the currently available prosthesis

\begin{tabular}{|c|c|c|c|c|}
\hline \multirow[t]{2}{*}{ Anatomy } & \multicolumn{2}{|c|}{ CoreValve revalving system } & \multicolumn{2}{|c|}{ Edwards SAPIEN XT } \\
\hline & $26 \mathrm{~mm}$ & $29 \mathrm{~mm}$ & $23 \mathrm{~mm}$ & $26 \mathrm{~mm}$ \\
\hline \multicolumn{5}{|l|}{ Peripheral arteries and aorta } \\
\hline Iliofemoral artery diameter (mm) & $\geq 6(18 \mathrm{Fr})$ & & $\geq 6(18 \mathrm{Fr})$ & $\geq 6.5(19 \mathrm{Fr})$ \\
\hline \multicolumn{5}{|l|}{ Tortuosity } \\
\hline \multicolumn{5}{|l|}{ Calcification } \\
\hline \multicolumn{5}{|l|}{ Aortic valve } \\
\hline \multicolumn{5}{|l|}{ Anatomy } \\
\hline \multicolumn{5}{|l|}{ Calcification } \\
\hline Annular diameter (mm) & $20-23$ & $24-27$ & $18-22$ & $21-25$ \\
\hline \multicolumn{5}{|l|}{ Aortic root } \\
\hline Sinus of Valsalva diameter (mm) & $\geq 27$ & $\geq 28$ & NA & \\
\hline Sinotubular junction diameter (mm) & $\leq 40$ & $\leq 43$ & NA & \\
\hline Ascending aorta diameter (mm) & $\leq 43$ & & NA & \\
\hline $\begin{array}{l}\text { Height of the coronary ostia from the } \\
\text { aortic annular plane (mm) }\end{array}$ & $\geq 10$ & & $\geq 8$ & \\
\hline Left ventricular septal thickness $(\mathrm{mm})$ & $<17$ & & NA & \\
\hline Coronary artery anatomy & $\begin{array}{r}\text { Not in sever } \\
\text { not amena }\end{array}$ & $\begin{array}{l}1 \text { coronary lesions } \\
\text { ascularization }\end{array}$ & $\begin{array}{l}\text { Not in seve } \\
\text { not amena }\end{array}$ & $\begin{array}{l}\text { al coronary lesions } \\
\text { ascularization }\end{array}$ \\
\hline Intracardiac thrombus & Absent & & Absent & \\
\hline
\end{tabular}
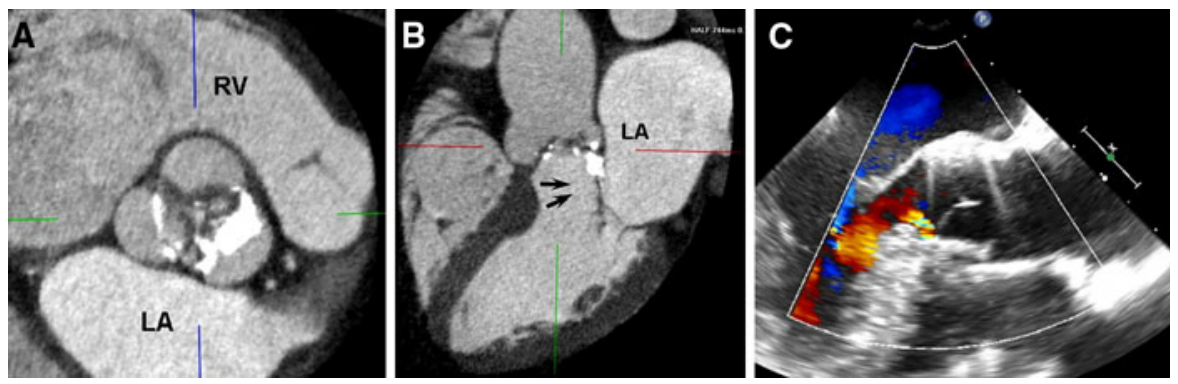

Fig. 1 Aortic valve calcification assessed using multidetector row computed tomography $(M D C T)$ : implications for transcatheter aortic valve implantation. a shows a calcified tricuspid aortic valve with bulky calcification mainly in the left cusp, left-right commissure and extending to the base of the anterior mitral valve leaflet (indicated by arrows in b). Following TAVI, paravalvular leak was observed with colour Doppler transesophageal echocardiography in the long-axis view

causing ventricular displacement of the device at the time of deployment [15]. Hence, appreciation of the extent and location of calcification, more precisely measured using MDCT, may help to anticipate and thus avoid potential procedural complications. (c) that coincided with the location of bulky calcification at the left-right commissure on MDCT (in a). In this example, the bulky calcified cusp and commissure might pose resistance during transcatheter prosthesis deployment, resulting in subsequent paravalvular leak (arising from the gap between the prosthesis and native valve). $L A$ left atrium, $R V$ right ventricle

Besides its implications on the procedural outcome of TAVI, the assessment of aortic valve calcification can be a useful adjunct in the evaluation of AS severity during pre-procedural screening $[16,17]$. In a recent study, the degree of aortic valve calcification 
measured using MDCT was highly correlated with the hemodynamic severity of AS measured using echocardiography [16].

Accurate evaluation of the aortic valve annular dimension is key for appropriate selection of prosthesis size. This process is unique in percutaneous procedures as direct inspection of the valve is not possible. Currently, the Edwards SAPIEN XT device is available in two sizes: $23 \mathrm{~mm}$ valve for aortic annulus between 18 and $22 \mathrm{~mm}$ and $26 \mathrm{~mm}$ valve for aortic annulus between 21 and $25 \mathrm{~mm}$. Similarly, the Medtronic CoreValve system has two sizes: $26 \mathrm{~mm}$ valve for aortic annulus between 20 and $23 \mathrm{~mm}$ and $29 \mathrm{~mm}$ valve for aortic annulus between 24 and $27 \mathrm{~mm}$ (Table 1) [18]. This step is critical as inaccurate sizing will result in undesirable periprocedural consequences such as prosthesis migration, significant aortic regurgitation (if undersized) or rupture of the aortic root (if oversized). In most centers, the measurement of the aortic annular diameter is performed using 2D echocardiography. In clinical practice, it is widely recognised that the aortic annulus is defined at the lowest attachment point of the aortic valve leaflets within the left ventricle (LV), forming a virtual ring [19]. As previously shown, this functional ring is not circular but oval in shape (Fig. 2), and is more accurately visualized with $3 D$ imaging techniques [20, 21]. In a recent study of patients undergoing open aortic valve surgery, Smíd et al. [22] compared pre-operative measurements of the aortic annulus using MDCT, magnetic resonance imaging (MRI) and 2D transesophageal echocardiography. Using intra-operative direct measurement as the reference, the accuracy of pre-operative measurement using either MDCT or MRI were superior compared to echocardiographic measurement, highlighting the high precision achievable with MDCT [22]. This is partly due to the high spatial resolution of MDCT which permits improved visualization of the aortic valve. Another explanation is that the anatomical planes of measurement obtained with different modalities are not identical $[23,24]$. For example, the parasternal long-axis view for transthoracic or transesophageal echocardiography represents an oblique cut through the aortic annulus and provides a single annular dimension that usually does not correspond to a true anatomical diameter measurement (either the maximum or minimum diameter visualised on MDCT) (Fig. 2).

Given that the aortic annulus is oval in shape, only 3D imaging techniques can provide the most accurate assessment of the aortic annulus dimension. As such, MDCT permits reconstructions in unlimited planes, allowing multiple measurements of the aortic annulus: minimum $\left(D_{\min }\right)$, maximum $\left(D_{\max }\right)$, mean $\left(D_{\text {mean }}=\right.$ $\left.\left[D_{\min }+D_{\max }\right] / 2\right)$ diameters and cross-sectional areas (Fig. 2) [21, 25]. Depending on how the aortic annulus is measured, the selection of the prosthesis size may differ. For example, in a recent study of 75 patients with severe AS undergoing MDCT as part of procedural planning for TAVI, Schultz and co-workers [25] showed that ineligibility for the currently available Medtronic CoreValve system differed substantially if $D_{\min }$ or $D_{\max }$ were used. Thus, 26 or $39 \%$ of patients would not qualify for TAVI with Medtronic CoreValve system due to too small or too large annular dimensions, respectively. In 50 patients who subsequently received the Medtronic
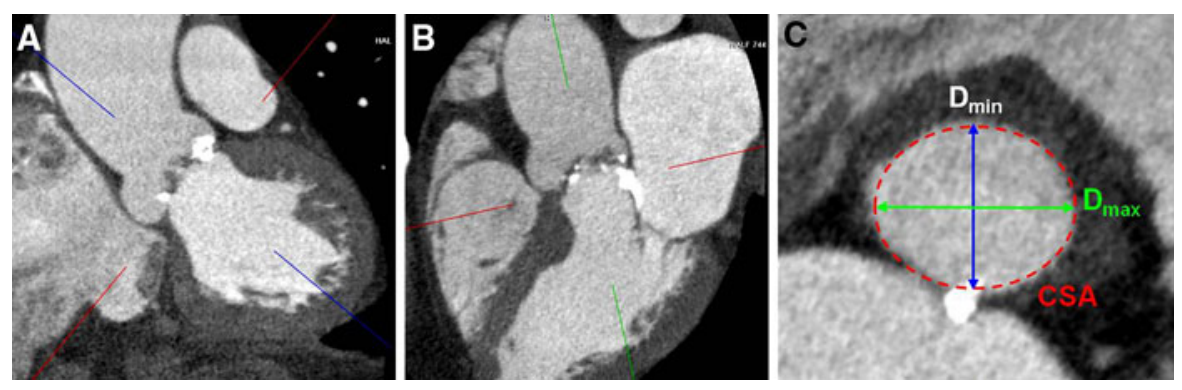

Fig. 2 Aortic valve annular dimensions. Multidetector row computed tomography ( $M D C T)$ permits excellent visualization of the oval-shaped aortic annulus with correct alignment of the orthogonal multiplane reformation planes (a and b). The correct aortic annular plane is defined at the lowest attachment point of all the three valve leaflets (c) and multiple measurements of the aortic annulus can be made: minimum $\left(D_{\text {min }}\right)$, maximum $\left(D_{\text {max }}\right)$, mean $\left(D_{\text {mean }}=\left[D_{\text {min }}+D_{\max }\right] / 2\right)$ diameters and cross-sectional areas (CSA) 
CoreValve prosthesis, the sizing based on $D_{\text {mean }}$ had the best agreement with the operator choice $(n=37$, $74 \%$ ), whereas the agreement with the operator choice was only 44 or $32 \%$ if $D_{\min }$ or $D_{\max }$ were used, respectively [25]. For the Edwards SAPIEN prosthesis, Messika-Zeitoun et al. [26] reported that using $D_{\text {mean }}$, as measured by MDCT, would have changed the prosthesis size in $38 \%$ of patients. Prospective studies examining the value of different imaging modalities in sizing of prosthesis and its immediate impact on procedural outcomes are lacking but are important to establish the gold standard methodology to size the aortic valve annulus and select the prosthesis size.

In addition, the assessment of the dimensions of the sinus of Valsalva, sinotubular junction and ascending aorta is an essential step in the pre-procedural evaluation. Using the "center-line approach" and reformations of the aortic root and ascending aorta, MDCT permits accurate measurement of these dimensions. This information is critical especially in patients undergoing the self-expandable device implantation as a dilated aortic root/ascending aorta is currently a contraindication (Table 1) [6].

Next, the 3D analysis of MDCT permits comprehensive and detailed evaluation of the spatial relationship of the aortic valve with the surrounding structures. In particular, the information on the height of the coronary ostia relative to the aortic annular plane is important to ensure patency of the coronary arteries following ballooning and deployment of the transcatheter prosthesis (Fig. 3). Currently, a minimum distance of $10 \mathrm{~mm}$ is recommended for both devices [18]. In addition, MDCT is an ideal modality to measure the length of the valvular leaflets as it can potentially increase the risk of coronary occlusion, notably in patients with bulky calcification of the aortic leaflets (Fig. 3b) [14]. However, this requirement may vary from individual to individual as the final position of the prosthesis depends on the interaction between the prosthesis and the aortic annulus [15].
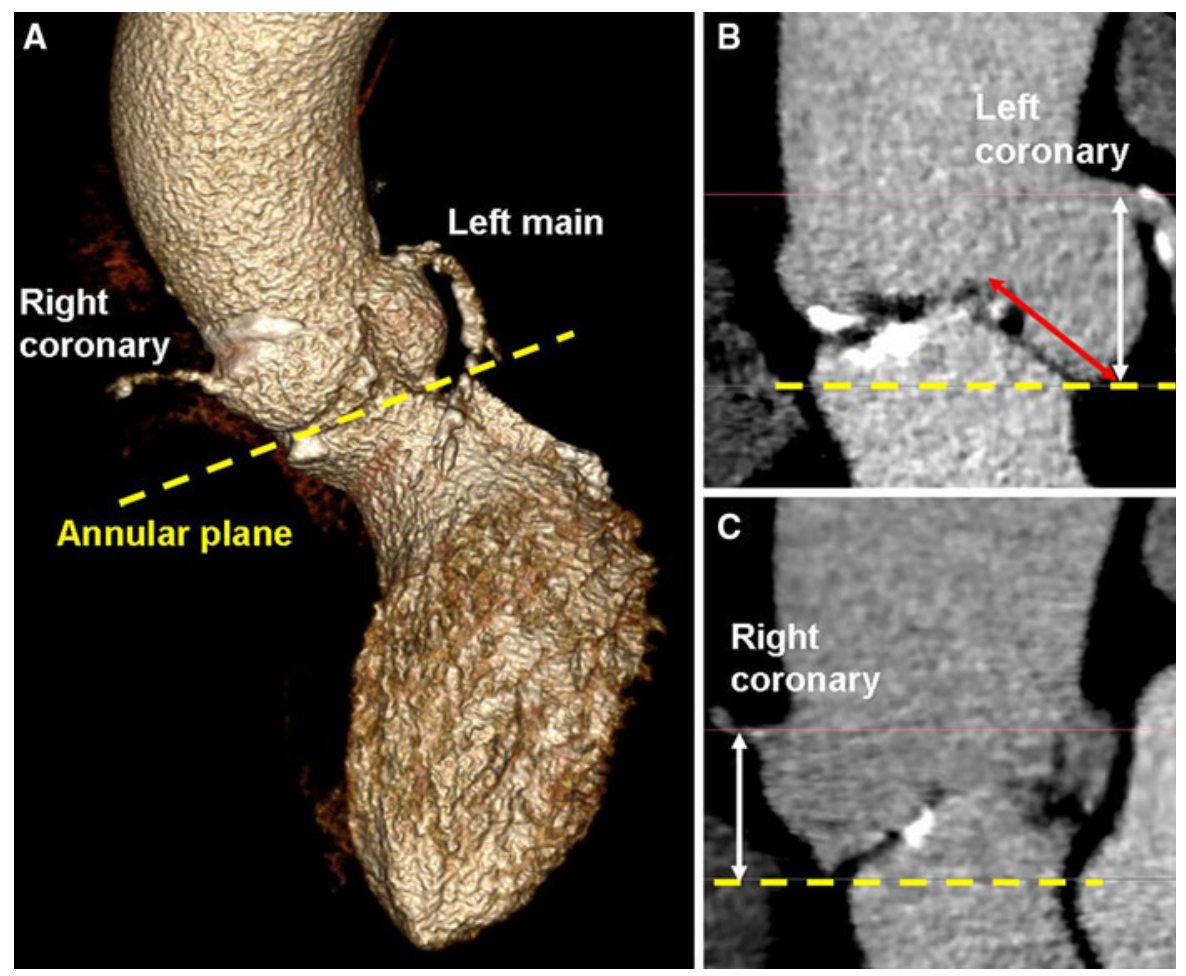

Fig. 3 Assessment of the height of the coronary ostia relative to the aortic annular plane. Multidetector row computed tomography $(M D C T)$ permits accurate orientation of the aortic annular plane (a) and precise measurement of the distance between the left and right coronary ostia and the annular plane (b and c). In addition, the length of the valvular leaflet, measured from the aortic annulus to the cusp tip, can be obtained on MDCT (red arrow in b) 
Finally, evaluation of the peripheral access artery and aortic anatomy is fundamental to plan the procedural strategy: retrograde (through a transarterial approach, transfemoral or transsubclavian) or antegrade (through a transapical approach). Although recent advances have allowed for a lower crimped profile delivery device, the transfemoral approach for self- or balloon-expandable systems can only be considered when the minimal diameter of the iliofemoral vessel is $\geq 6 \mathrm{~mm}$ (to accommodate a $18 \mathrm{~F}$ sheath size) [27]. As adequate access is one of the most important determinants of procedural success [28], the decision for selecting the transfemoral approach relies on the precise measurement of vessel dimension, tortuosity and calcification of the peripheral arteries and aorta [6]. In the recent multicenter SOURCE registry of 1,038 patients who received a balloon-expandable prosthesis, the rate of vascular complications with the transfemoral approach was higher $(22.9 \%$ vs. $4.7 \%)$ when compared with the transapical approach, highlighting the need for careful patient selection, to select the most suitable procedural approach to avoid procedural-related vascular complications [29].

Although conventional angiography is the reference method to assess the luminal diameter, tortuosity and calcification of the peripheral arteries and aorta [6], true cross-sectional diameters and areas are better visualized on MDCT. Typically, the curved multiplanar reformation planes (MPR), using the "center-line approach", permits reconstruction of the curved planes, following the course of the vessel regardless of its tortuous course. Ideally, the precise measurement of the vascular structures should be obtained from the axial view, perpendicular to the long axis of the vessel (Fig. 4c, d). In the presence of calcification, the blooming effect has to be brought to a low level when these measurements are being performed.

With the 3D volume rendering images, current MDCT techniques permit rotation and display to best define the total number and severity of angulations along the vessel of interest. Post-processing imaging software is available to detect all angulations along the vessel automatically and allows precise measurement of the severity of angulations (Fig. 4e), which helps to systematically quantify the extent of tortuosity in patients who are being considered for a transfemoral procedure. In addition, MDCT can accurately delineate the location and the extent of calcification along the vessel. This is particularly important in case of a tortuous vessel as significant calcification does not allow straightening of the vessel during advancement of the sheath and should prompt the consideration of an alternative access (either the transapical or transsubclavian approach). Furthermore, severe calcification at the bifurcation of the iliac vessels may become a concern for the transfemoral approach as it may restrict sheath advancement and increase the risk of vessel perforation or dissection. In a recent study by Kurra et al. [30] that examined 100 patients who were considered for TAVI, as many as $35 \%$ of patients had unsuitable iliofemoral anatomy defined as one of the following: minimal diameter of the iliofemoral vessel $<8 \mathrm{~mm}$ (a requirement for the older generation delivery system), $>60 \%$ circumferential calcification at the external-internal iliac bifurcation or severe angulation $<90^{\circ}$. Among those with MDCT criteria of unsuitable anatomy $(n=35), 5$ patients proceeded with transfemoral-TAVI. Of these 5 patients, 2 (40\%) had vascular complications requiring surgical intervention [30].

Besides the anatomical requirement of the iliofemoral arteries, the pre-procedural assessment of the aorta and its lumen is necessary as it may guide the approach of TAVI. Presence of bulky atherosclerosis of the aorta, a porcelain aorta, a transverse course of the ascending aorta or a previous aorto-femoral bypass is a contraindication for the transfemoral approach [6]. Fluoroscopy, commonly used to evaluate the luminal diameter of the peripheral arteries, does not allow accurate assessment of arterial wall disease or atherosclerotic plaques. In contrast, MDCT provides a comprehensive evaluation including arterial luminal diameter and wall assessment. The presence of extensive aortic atherosclerosis as detected with MDCT may preclude the transfemoral approach due to the increased risk of cerebrovascular events during manipulation of the catheters along the diseased aorta.

Although suitable vessel anatomy is the key consideration in the transfemoral approach, other aspects need to be considered, which may favour one approach over another. For instance, pericardial calcification, a deformed chest wall anatomy or severe pulmonary disease may make the transapical approach unsuitable and MDCT is helpful to provide such information. 


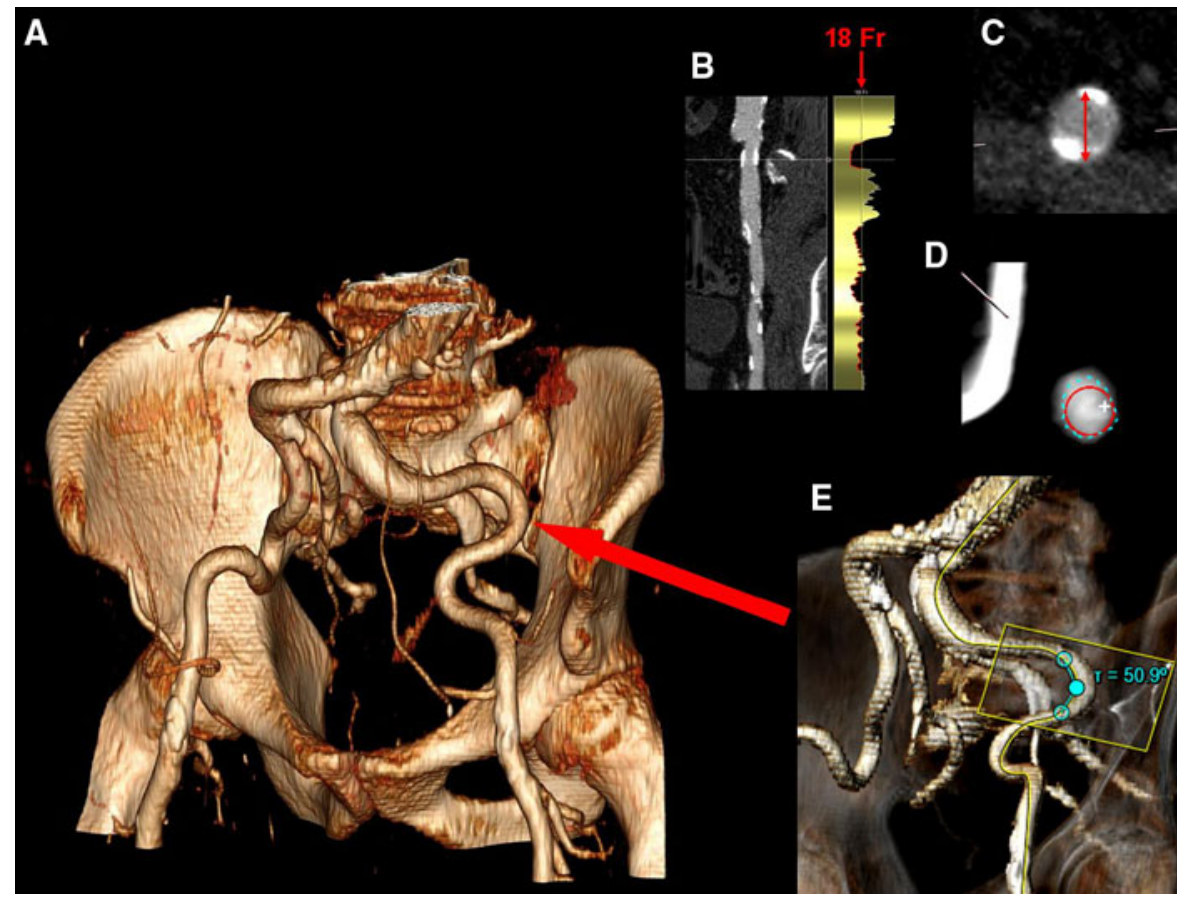

Fig. 4 Evaluation of peripheral arteries with multidetector row computed tomography $(M D C T)$. a shows an example of infrarenal aorta, iliac and femoral arteries in a 3-dimensional volume rendering view. Using the center-line approach, the curved multiplanar reformation $(M P R)$ permits reconstruction of the curved planes, following the course of the vessels. Subsequently, the true cross-sectional internal diameter and area of the iliac artery can be measured from the double oblique transverse view in (c and d). With the current MDCT post-processing imaging software (3mensio Valves ${ }^{\text {TM }}$, version 4.2., 3mensio Medical Imaging BV, Bilthoven, The Netherlands), the minimum diameter threshold required for the currently available transfemoral devices is $6 \mathrm{~mm}(18 \mathrm{Fr})$ and

\section{Planning of interventional access planes}

Pre-operative CT has been demonstrated to accurately predict the location of the aortic valve and ascending aorta relative to the chest wall in patients undergoing a minimally invasive approach for aortic valve replacement [31]. Several groups have reported that 3D data obtained from MDCT can help to predict the fluoroscopic projections that are optimal for TAVI procedures [32-34]. An ideal angiographic plane (the so called "implanter's view") should be the projection that aligns all three aortic cusps in a straight line, perpendicular to the aortic valve plane (Fig. 5) [15]. A recent study by Gurvitch et al. [32] compared 2 groups of patients who underwent TAVI with (20 patients) and without (20 patients) this minimum requirement is simultaneously displayed sideby-side in the curved MPR views (b). Therefore, the presence of a minimal luminal diameter of the iliofemoral arteries $<6 \mathrm{~mm}$ does not favor the transfemoral approach. In contrast, the example in $\mathbf{d}$ shows a vessel with a minimal luminal diameter $>6 \mathrm{~mm}$, as indicated by the dotted green circle which is larger than the size of a simulated 18 Fr sheath (in solid red circle). In addition, the 3-dimensional reconstruction volume rendering technique of MDCT allows rotations and displays the tortuous course of the iliofemoral arteries. e gives the precise measurement of one of the angulations seen in the left external iliac artery $\left(51^{\circ}\right)$, rendering it unsuitable for the transfemoral approach

pre-procedural MDCT. When MDCT information was available to guide the fluoroscopic projection angle, an excellent or satisfactory final implant projection was achieved in $90 \%$ of cases $(n=18)$, as compared to only $65 \%$ of cases $(n=13)$ when pre-procedural MDCT was not available [32].

With regard to the transapical approach, the relation of the LV apex and the aortic annulus plane (the so called "ventriculo-aortic angle") can be important (Fig. 6). Recently, the first-in-man series of a new self-expanding prosthesis implantation via the transapical approach was reported in 30 patients [35]. The initial experience highlighted the importance of this ventriculo-aortic angle, which may pose as a challenge during the introduction of a straight and rigid delivery system (via the LV apex) which 

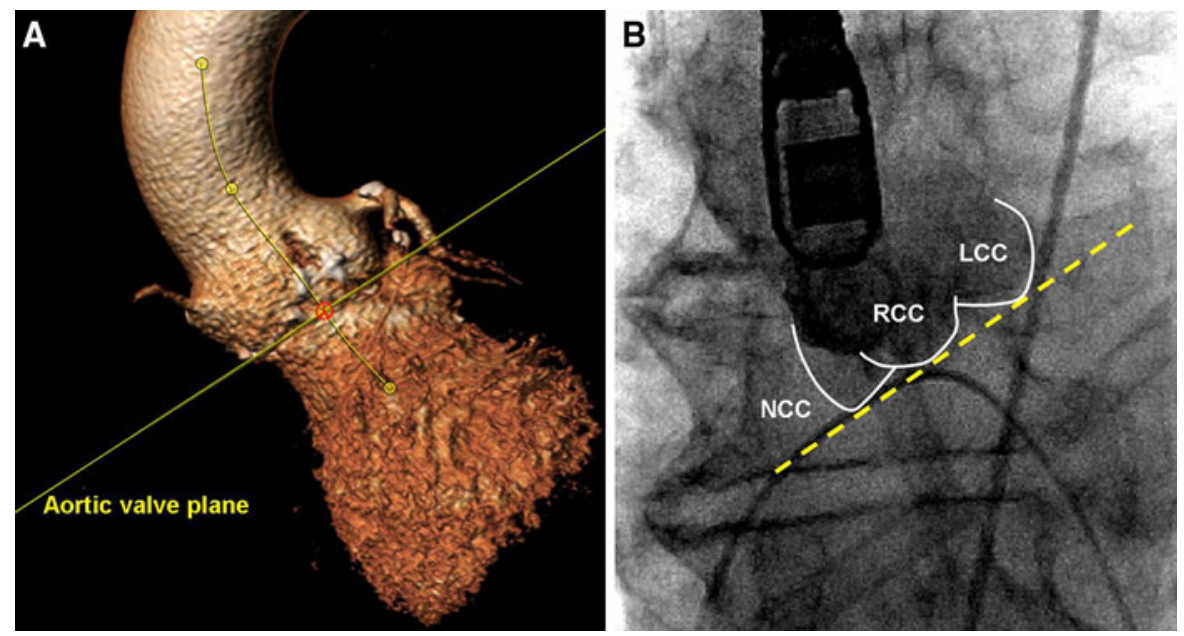

Fig. 5 Planning of angiographic planes. Using reformation reconstruction of multidetector row computed tomography $(M D C T)$, the appropriate aortic valve plane for transcatheter aortic valve implantation can be anticipated (a). The ideal angiographic projection should be one that aligns all the three aortic cusps in a straight line, perpendicular to the aortic valve plane (b). LCC left coronary cusp, NCC non-coronary cusp, $R C C$ right coronary cusp

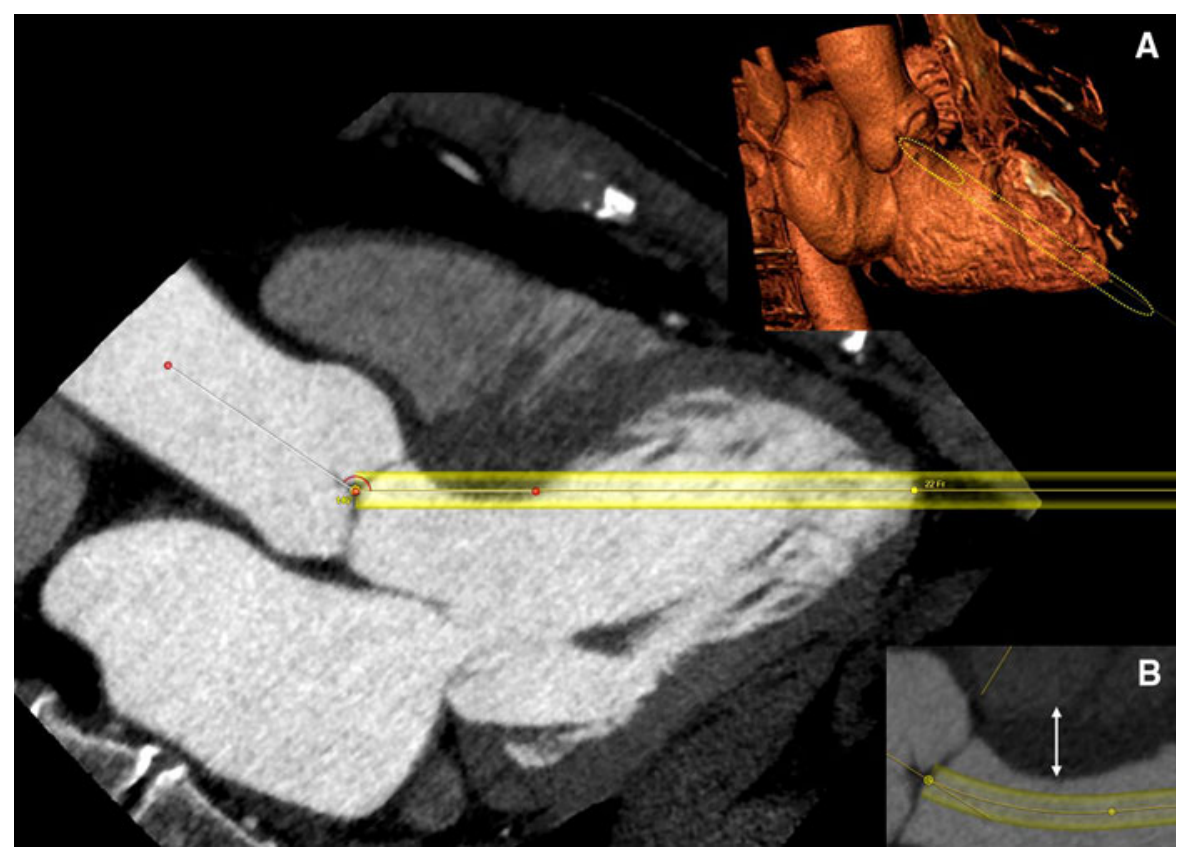

Fig. 6 Assessment of the left ventricular geometry may be of relevance in the planning of the transapical approach. The relation of the left ventricular apex and the aortic annulus valve plane (the so called "ventriculo-aortic angle") can be reliably measured on multidetector row computed tomography $(M D C T)$. Insert a shows the direction of a simulated delivery

may not be able to conform to this angle, subjecting patients to a risk of aortic dissection when the device is advanced into the ascending aorta [35]. Novel 3D system through the left ventricular apex, towards the aortic valve. In addition, the thickness of the left ventricular septum wall can be measured on MDCT (arrow in insert b). Postprocessing imaging software (3mensio Valves ${ }^{\mathrm{TM}}$, version 4.2., 3mensio Medical Imaging BV, Bilthoven, The Netherlands)

based post-processing imaging software is currently available to permit direct visualization of this ventriculo-aortic relationship to aid in the planning for the 
transapical approach. This allows the anticipation of the angulation required during insertion of the apical sheath and the delivery system, towards the direction of the aortic valve and the ascending aorta (Fig. 6). In addition, the measurement of the LV septal wall thickness with MDCT is important (Fig. 6b) as the presence of severe sigmoid basal septum may prevent stable positioning of the deployed prosthesis (Table 1).

\section{Other factors to be considered before TAVI}

In addition to the aforementioned considerations, additional factors need to be evaluated before TAVI, which completes the pre-procedural screening. The evaluation of coronary artery anatomy is mandatory as the presence of significant coronary artery disease needs to be revascularized. Current position statement does not recommend TAVI in patients with severe proximal coronary stenoses not amenable to percutaneous coronary interventions [6]. Although MDCT has shown its diagnostic accuracy in the evaluation of coronary artery disease [36], the prevalence of coronary atherosclerosis in the elderly population may limit its accuracy in detecting significant coronary artery stenoses. Therefore, invasive coronary angiography remains the reference modality to evaluate the coronary anatomy in this highly selected group of patients [6].

Finally, LV dimensions, function and the presence of concomitant mitral regurgitation (MR) need to be evaluated before TAVI. This information is usually available from the standard echocardiography, which is still the initial imaging modality in patients scheduled for TAVI. However, in patients with poor acoustic window, MDCT allows assessment of LV dimensions and function using ECG-gating. More importantly, it permits detection of intracardiac thrombus, which is an established contraindication for TAVI (Table 1) [6].

\section{Transcatheter mitral valve repair procedures}

Mitral valve repair is the treatment of choice for patients with symptomatic MR [4]. Advances in surgical techniques have led to improved clinical results in young and elderly patients [37]. However, associated comorbidities and low LV ejection fraction increase the operative morbidity and mortality risks in the elderly population and may lead to non-referral or denial for surgery in as many as $50 \%$ of the patients with symptomatic severe MR [38].

Transcatheter-based and minimally invasive surgical therapies have been developed over the last years. Several therapeutic options are now available for patients with symptomatic severe MR and high operative risk. These percutaneous techniques can be classified as leaflet-based (edge-to-edge repair, Mitraclip device [Abbott Vascular, Structural Heart, Menlo Park, CA, USA]), coronary sinus or mitral annulus-based (Carillon, Monarc or Viacor devices and Quantum cor and Mitralign devices, respectively) and LV-based (Coapsys device [Myocor, Maple Grove, MN]). The EVEREST II trial has shown the feasibility, safety and efficacy of the Mitraclip device in reducing MR and improving clinical symptoms [39]. In addition, initial experiences with devices designed to reduce the mitral annulus perimeter and improve the mitral leaflet coaptation (the AMADEUS and the EVOLUTION trials) demonstrated that transcatheter mitral restrictive annuloplasty approaches may be a feasible alternative to surgery in selected patients [40-42]. Furthermore, the results of the RESTORE-MV trial showed that patients with functional MR benefited from ventricular reshaping with the Coapsys device, with significant improvement in clinical symptoms and survival [43].

The feasibility and efficacy of these transcatheterbased or minimally invasive surgical therapies rely on the presence of suitable valve and LV anatomy and geometry. Evaluation of the underlying mechanism of MR is crucial to select the most appropriate transcatheter based therapy (Table 2). In brief, MR can be divided into organic or primary MR when the mitral valve itself is diseased (i.e. Barlow's disease, healed infective endocarditis) and secondary or functional MR when the mitral valve is anatomically normal but a remodeled and dysfunctional LV prevents adequate coaptation of the mitral leaflets. Two-dimensional and recently, 3D transesophageal echocardiography are the mainstay imaging techniques used in surgical decision-making (mitral valve repair or replacement). However, the high spatial resolution of MDCT permits accurate assessment of the anatomy, geometry and spatial relationships of the mitral valve complex and thus provides important information for selecting candidates for these therapies. 
Table 2 Transcatheter-based mitral valve repair techniques

\begin{tabular}{llll}
\hline Mitral valve repair technique & Device & Trial & Indication \\
\hline Leaflet repair & MitraClip & EVEREST I & Organic mitral valve regurgitation \\
& & EVEREST II & Functional mitral valve regurgitation \\
Coronary sinus-based annuloplasty & Carillon & AMADEUS & Functional mitral valve regurgitation \\
& Monarc & EVOLUTION & \\
Direct LV remodelling & PTMA & PTOLEMY-1 & \\
\hline
\end{tabular}

MDCT before mitral leaflet repair

The Mitraclip device is delivered to the mitral valve via percutaneous femoral venous transseptal puncture and creates a double-orifice valve, resulting in improved mitral leaflet coaptation and MR reduction. After crossing the interatrial septum, the delivery system is steered toward the mitral valve plane and the clip is aligned perpendicular to the mitral leaflets and centered in the area with the largest effective regurgitant orifice area. Afterwards, the delivery system is advanced into the LV and the arms of the clip are opened for subsequent grasping and coaptation of the leaflets at the targeted scallops.

Transesophageal echocardiography plays a central role in pre-procedural screening and procedural guidance during the intervention. Current 3D transesophageal echocardiography permits visualization of the mitral valve from multiple perspectives, orientation of the MPR to localize the largest regurgitant orifice and accurate characterization of the underlying mechanism of MR. Similarly, MDCT enables accurate $3 \mathrm{D}$ visualization of the mitral leaflets and detailed evaluation of the anatomic criteria essential in percutaneous Mitraclip implantation:

- Central regurgitant jet, located at the central scallops of the anterior (A2) and posterior (P2) mitral leaflets.

- Coaptation length $\geq 2 \mathrm{~mm}$ and coaptation depth $\leq 11 \mathrm{~mm}$ (for functional MR).

- flail gap $<10 \mathrm{~mm}$ and flail width $<15 \mathrm{~mm}$ (for organic MR).

Multidetector row computed tomography data reconstructed in smaller (such as 5\%) increments throughout the RR interval provide high spatial resolution images with improved temporal resolution and enable identification of the systolic frame where mitral leaflet coaptation failure occurs.

In addition, MDCT provides information on the underlying mechanism of MR. For example, the diagnostic performance of MDCT to identify mitral valve prolapse was recently evaluated in a series of 53 patients [44]. The orientation of the MPR across the mitral valve plane provides the LV 4-, 2- and 3 -chamber apical views and enables localization of the mitral valve prolapse, billowing and flail leaflet (Fig. 7). The sensitivity, specificity, positive predictive value and negative predictive value of MDCT for diagnosis of mitral valve prolapse were 96, 93, 93 and $96 \%$, respectively [44]. Furthermore, the assessment of the mitral valve geometry and the measurement of the tenting heights (coaptation depth) and leaflet angles can be accurately performed in patients with functional MR [45]. The orientation of the orthogonal MPR across the modified short-axis view of the mitral valve provides the 4-chamber view at the anterolateral (A1-P1), central (A2-P2) and posteromedial (A3-P3) levels (Fig. 8). In a series of 67 heart failure patients, including 29 patients with significant functional MR, the mitral valve geometry was evaluated with MDCT [45]. In patients with significant MR, the maximum tenting height and tethering of the posterior mitral leaflet were located at the central and posteromedial levels. The knowledge of these data beforehand permits accurate planning of the procedural strategy and may result in a significant shortening of fluoroscopy and procedure timings.

MDCT before coronary sinus annuloplasty

Coronary sinus-based mitral annuloplasty devices have been designed to treat functional MR percutaneously. Two anchors or stents connected by a bridge 


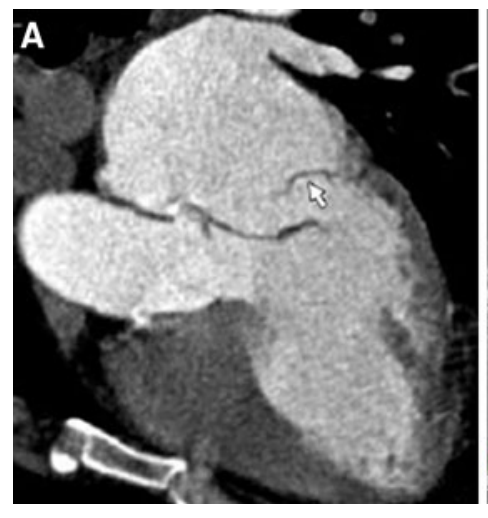

Fig. 7 Evaluation of underlying mechanism of mitral regurgitation with multidetector row computed tomography $(M D C T)$. Mitral valve prolapse can be identified accurately with MDCT. a shows an example of a patient with prolapse of

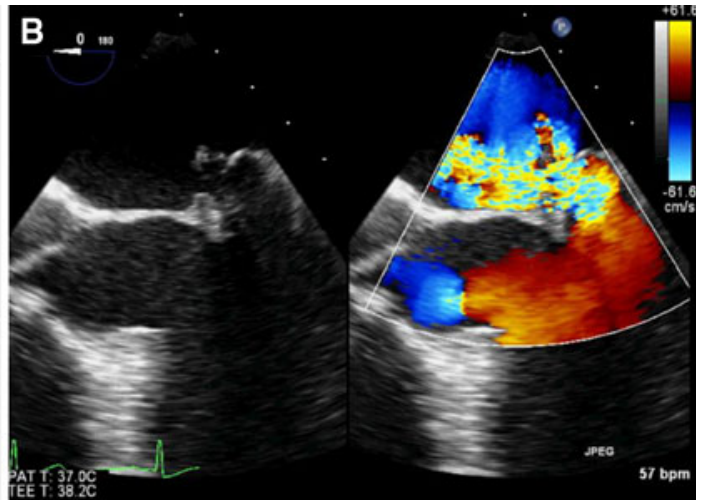

the posterior leaflet (arrow). Color Doppler echocardiography permits quantification of the regurgitant volume and the direction of the regurgitant jet (b). Modified with permission from Feutchner et al. [44]

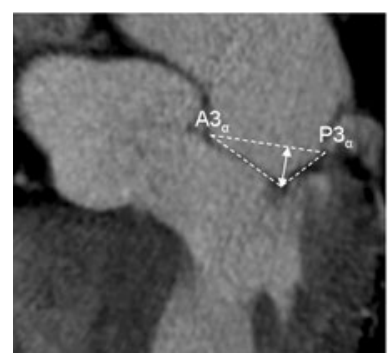

Fig. 8 Mitral valve geometry assessment with multidetector row computed tomography (MDCT) in functional mitral regurgitation. From the short-axis view of the mitral valve at the level of the mitral leaflets and commissures, the orthogonal

element are placed within the distal part of the coronary sinus or the great cardiac vein and in the coronary sinus ostium. The bridging connector constraints the coronary sinus and reduces the anteroposterior diameter of the mitral annulus, improving the coaptation of the mitral leaflets and reducing the MR. Data from the AMADEUS trial have demonstrated the feasibility, safety and efficacy of this therapy [42]. Out of 48 heart failure patients enrolled with significant functional MR, 30 patients received planes across the anterolateral $(A 1-P 1)$, central $(A 2-P 2)$ and posteromedial $(A 3-P 3)$ provide the apical views of the mitral valve apparatus and permits the measurement of the leaflet angles and tenting heights (arrows)

the Carillon device (Cardiac Dimensions, Inc., Kirkland, WA). At 6 months follow-up, significant reductions in regurgitant volume, effective orifice regurgitant area and vena contracta were observed, together with significant improvements in clinical status [40]. However, one of the main concerns of this therapy is the possibility of impingement of the epicardial coronary arteries. In $17 \%$ of implants, a significant arterial impingement involving the circumflex coronary artery was observed. In addition, an 
insufficient change in MR grade was observed in 4 patients. The variable position and course of the coronary sinus relative to the mitral annulus is one of the determinants of the efficacy of this therapy.

Multidetector row computed tomography provides useful information on the dimensions of the coronary sinus and its position relative to the mitral annulus and the circumflex coronary artery [46-48]. Combining the axial views and $3 \mathrm{D}$ volume renderings, MDCT permits evaluation of the feasibility and safety of coronary sinus-based mitral annuloplasty procedures (Fig. 9). Tops et al. [47] evaluated the dimensions, course and spatial relationships of the coronary sinus in 105 patients undergoing MDCT. In $90 \%$ of patients, the coronary sinus was superior to the mitral valve annulus with a distance that ranged between 1.4 and $16.8 \mathrm{~mm}$. Importantly, this distance was significantly larger in patients with heart failure as compared to controls $(6.2 \pm 3.4 \mathrm{~mm}$ vs. $4.4 \pm$ $3.4 \mathrm{~mm}, P<0.05)$. Therefore, in a significant number of patients the coronary sinus coursed along the posterior wall of the left atrium rather than along the mitral annular plane reducing the efficacy of this device to improve MR. In addition, in $68 \%$ of
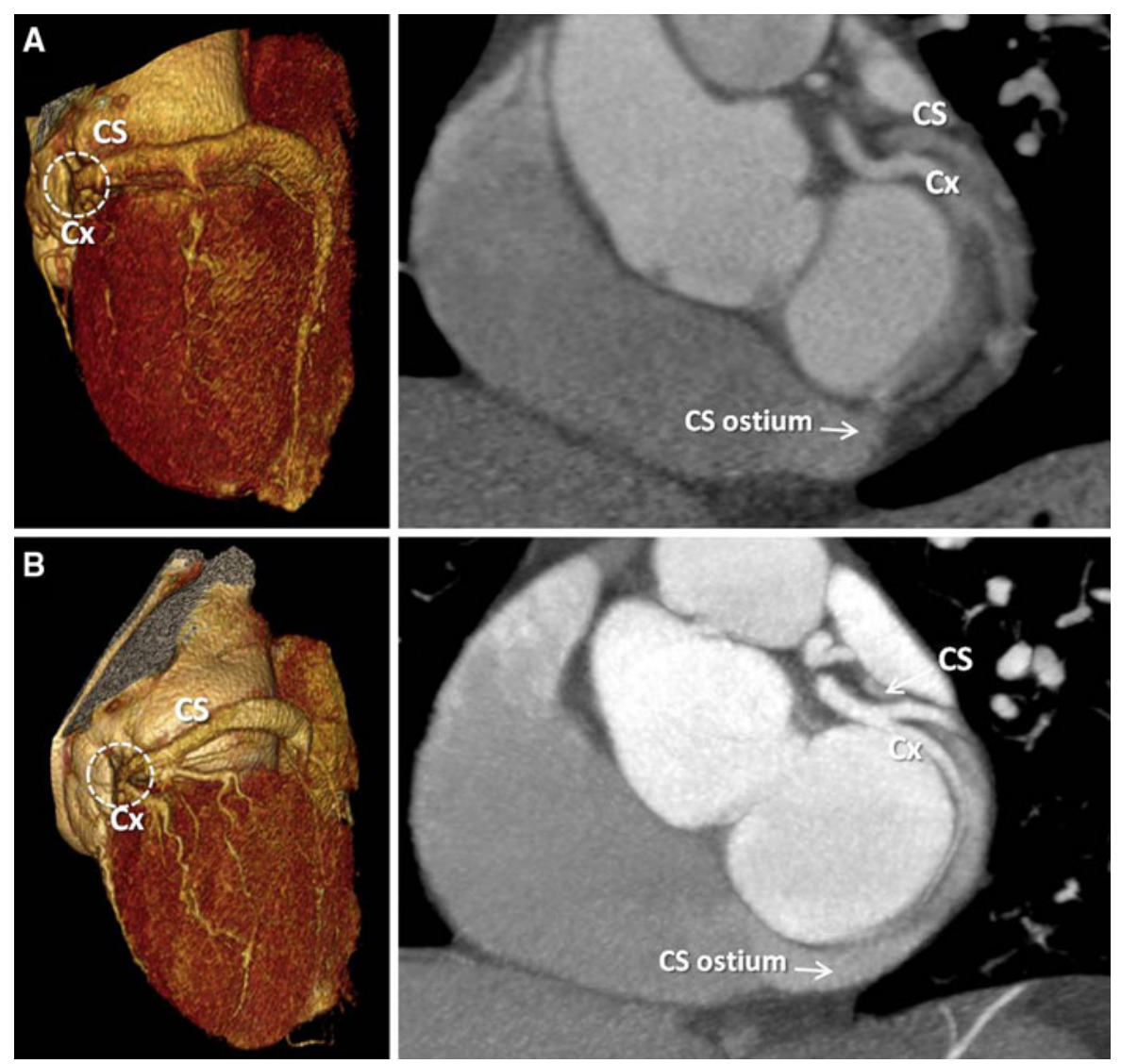

Fig. 9 Multidetector row computed tomography (MDCT) prior to coronary sinus-based mitral valve annuloplasty. Combination of 3-dimensional volume rendering and axial views of the mitral valve annulus permit assessment of the key anatomic relationships of the coronary sinus: its position relative to the mitral annular plane and the circumflex coronary artery. a shows an example of a patient with the coronary sinus properly aligned with the mitral annulus (as seen with the 3-dimensional volume rendering). However, at the level of the distal part of the coronary sinus, where the distal anchor is positioned, the circumflex coronary artery courses between the mitral annulus and the coronary sinus. The risk of coronary impingement in this example may contraindicate the procedure. In contrast, $\mathbf{b}$ shows an example where the coronary sinus courses superiorly to the posterior mitral annulus. The coronary sinus-based mitral annuloplasty may be less effective in this case, since the tension is applied to the posterior wall of the left atrium rather than the mitral annulus. In addition, there is a potential risk of circumflex coronary artery compromise as the distal part of the coronary sinus courses over the artery (arrow). $C S$ coronary sinus, $C X$ left circumflex artery 
patients, the circumflex coronary artery coursed between the coronary sinus and the mitral annulus, indicating an increased risk of arterial impingement during percutaneous coronary sinus-based annuloplasty. This information can be also obtained with MRI, a valuable alternative to MDCT in patients with severe renal dysfunction in whom the use of iodinated contrast may be contraindicated [49].

\section{MDCT before direct LV remodeling}

The RESTOR-MV trial evaluated the efficacy of the Coapsys device (Myocor, Inc., Maple Grove, MN) in reducing MR and improving clinical outcomes of heart failure patients with functional MR undergoing surgical revascularization [43]. This device is placed without the need of cardiopulmonary bypass and aims to reduce the mitral valve annular dimensions and correct the displacement of the papillary muscles. The anterior and posterior pads are positioned on the epicardial surface of the heart and the expanded polytetrafluoroethylene-coated subvalvular cord that connects both pads is tightened under echocardiographic guidance until significant reduction or elimination of MR is achieved [50]. Several anatomic and geometric criteria determine the eligibility for this procedure. Presence of structural abnormality of the mitral valve apparatus (i.e. leaflet prolapse, chordal rupture, mitral annular calcification or calcified leaflets) and LV end-diastolic diameter $>70 \mathrm{~mm}$ contraindicate this procedure. In addition, the potential interference with the papillary muscles or the inability to avoid main epicardial coronary arteries during device positioning may influence the feasibility of this treatment.

As previously mentioned, MDCT provides accurate characterization of the $L V$ dimensions, anatomy and location of the papillary muscles and location and extent of mitral valve apparatus calcification [45, 51]. Furthermore, the position of the anterior and posterior pads can be anticipated during pre-procedural screening, by visualizing the position of the main epicardial coronary arteries in the $3 \mathrm{D}$ volume renderings. Compared to the group of patients undergoing surgical coronary artery bypass grafting alone or in combination with mitral valve repair, the RESTOR-MV showed a significant improvement in MR, LV systolic function and survival of patients with ischemic heart failure and functional MR [43].

\section{Conclusions}

Accurate selection of patients who are candidates for a transcatheter-based valve repair/implantation technique results in high success rates and reduces the number of procedural complications. MDCT should play a central role in both aortic and mitral transcatheter-based interventions as it provides a comprehensive assessment of the anatomy prior to the procedure and helps to select the most appropriate procedural approach, making the procedure as safe as possible. In addition, the use of state of the art scanners and dose modulation MDCT protocols can potentially optimise the iodined-based contrast load and reduce the radiation exposure.

Conflict of interest Dr. See Hooi Ewe is financially supported by the Ministry of Health Training Scholarship, Singapore. Dr. Robert Klautz is a member of one of the advisory boards of Medtronic and receives research support from Edwards Lifesciences, St. Jude Medical and Medtronic. Dr. Martin J. Schalij receives grants from Biotronik, Boston Scientific \& Medtronic.

Open Access This article is distributed under the terms of the Creative Commons Attribution Noncommercial License which permits any noncommercial use, distribution, and reproduction in any medium, provided the original author(s) and source are credited.

\section{References}

1. Leon M, Smith C, Mack M et al (2010) Transcatheter aortic-valve implantation for aortic stenosis in patients who cannot undergo surgery. N Engl J Med 363:1597-1607

2. Mauri L, Garg P, Massaro JM et al (2010) The EVEREST II trial: design and rationale for a randomized study of the evalve mitraclip system compared with mitral valve surgery for mitral regurgitation. Am Heart J 160:23-29

3. Coeytaux RR, Williams JW, Gray RN et al (2010) Percutaneous heart valve replacement for aortic stenosis: state of the evidence. Ann Intern Med 153:314-324

4. Bonow RO, Carabello BA, Chatterjee K et al (2008) Focused update incorporated into the ACC/AHA 2006 guidelines for the management of patients with valvular heart disease: a report of the American College of Cardiology/American Heart Association Task Force on practice guidelines. J Am Coll Cardiol 52:e1-e142

5. Chiam PTL, Chao VTT, Tan SY et al (2010) Percutaneous transcatheter heart valve implantation in a bicuspid aortic valve. JACC Cardiovasc Interv 3:559-561

6. Vahanian A, Alfieri O, Al-Attar N et al (2008) Transcatheter valve implantation for patients with aortic stenosis: a position statement from the European Association of Cardio-Thoracic Surgery (EACTS) and the European 
Society of Cardiology (ESC), in collaboration with the European Association of Percutaneous Cardiovascular Interventions (EAPCI). Eur Heart J 29:1463-1470

7. Zegdi R, Lecuyer L, Achouh P et al (2010) Increased radial force improves stent deployment in tricuspid but not in bicuspid stenotic native aortic valves. Ann Thorac Surg 89:768-772

8. Tanaka R, Yoshioka K, Niinuma H et al (2010) Diagnostic value of vardiac CT in the evaluation of bicuspid aortic stenosis: comparison with echocardiography and operative findings. Am J Roentgenol 195:895-899

9. Alkadhi H, Leschka S, Trindade PT et al (2010) Cardiac CT for the differentiation of bicuspid and tricuspid aortic valves: comparison with echocardiography and surgery. Am J Roentgenol 195:900-908

10. Delgado V, Ng ACT, Van de Veire NR et al (2010) Transcatheter aortic valve implantation: role of multidetector row computed tomography to evaluate prosthesis positioning and deployment in relation to valve function. Eur Heart J 31:1114-1123

11. John D, Buellesfeld L, Yuecel S et al (2010) Correlation of device landing zone calcification and acute procedural success in patients undergoing transcatheter aortic valve implantations with the self-expanding CoreValve prosthesis. JACC Cardiovasc Interv 3:233-243

12. Koos R, Mahnken AH, Dohmen G et al (2010) Association of aortic valve calcification severity with the degree of aortic regurgitation after transcatheter aortic valve implantation. Int J Cardiol (in press)

13. Zegdi R, Ciobotaru V, Noghin M et al (2008) Is it reasonable to treat all calcified stenotic aortic valves with a valved stent? Results from a human anatomic study in adults. J Am Coll Cardiol 51:579-584

14. Webb JG, Chandavimol M, Thompson CR et al (2006) Percutaneous aortic valve implantation retrograde from the femoral artery. Circulation 113:842-850

15. Kapadia SR, Schoenhagen P, Stewart W et al (2010) Imaging for transcatheter valve procedures. Curr Probl Cardiol 35:228-276

16. Cueff C, Serfaty JM, Cimadevilla C et al (2010) Measurement of aortic valve calcification using multislice computed tomography: correlation with haemodynamic severity of aortic stenosis and clinical implication for patients with low ejection fraction. Heart (in press)

17. Messika-Zeitoun D, Aubry MC, Detaint D et al (2004) Evaluation and clinical implications of aortic valve calcification measured by electron-beam computed tomography. Circulation 110:356-362

18. Delgado V, Ewe S, Ng A et al (2010) Multimodality imaging in transcatheter aortic valve implantation: key steps to assess procedural feasibility. EuroIntervention 6:643-652

19. Piazza N, De Jaegere P, Schultz C et al (2008) Anatomy of the aortic valvar complex and its implications for transcatheter implantation of the aortic valve. Circ Cardiovasc Interv 1:74-81

20. Ng ACT, Delgado V, van der Kley F et al (2010) Comparison of aortic root dimensions and geometries before and after transcatheter aortic valve implantation by 2 - and 3-dimensional transesophageal echocardiography and multislice computed tomography. Circ Cardiovasc Imaging 3:94-102
21. Tops LF, Wood DA, Delgado V et al (2008) Noninvasive evaluation of the aortic root with multislice computed tomography: implications for transcatheter aortic valve replacement. JACC Cardiovasc Imaging 1:321-330

22. Smid M, Ferda J, Baxa J et al (2010) Aortic annulus and ascending aorta: comparison of preoperative and periooperative measurement in patients with aortic stenosis. Eur J Radiol 74:152-155

23. Schultz C, Moelker A, Tzikas A et al (2010) Cardiac CT: necessary for precise sizing for transcatheter aortic implantation. EuroIntervention 6(Suppl G):G6-G13

24. Tuzcu EM, Kapadia SR, Schoenhagen P (2010) Multimodality quantitative imaging of aortic root for transcatheter aortic valve implantation: more complex than it appears. J Am Coll Cardiol 55:195-197

25. Schultz CJ, Moelker A, Piazza N et al (2010) Three dimensional evaluation of the aortic annulus using multislice computer tomography: are manufacturer's guidelines for sizing for percutaneous aortic valve replacement helpful? Eur Heart J 31:849-856

26. Messika-Zeitoun D, Serfaty JM, Brochet E et al (2010) Multimodal assessment of the aortic annulus diameter: implications for transcatheter aortic valve implantation. J Am Coll Cardiol 55:186-194

27. Webb J, Cribier A (2010) Percutaneous transarterial aortic valve implantation: what do we know? Eur Heart J (in press)

28. Webb JG, Altwegg L, Masson JB et al (2009) A new transcatheter aortic valve and percutaneous valve delivery system. J Am Coll Cardiol 53:1855-1858

29. Thomas M, Schymik G, Walther T et al (2010) Thirty-day results of the SAPIEN aortic bioprosthesis European outcome (SOURCE) registry. A European registry of transcatheter aortic valve implantation using the Edwards SAPIEN valve. Circulation 122:62-69

30. Kurra V, Schoenhagen P, Roselli EE et al (2009) Prevalence of significant peripheral artery disease in patients evaluated for percutaneous aortic valve insertion: preprocedural assessment with multidetector computed tomography. J Thorac Cardiovasc Surg 137:1258-1264

31. Ammar R, Porat E, Eisenberg DS et al (1998) Utility of spiral CT in minimally invasive approach for aortic valve replacement. Eur J Cardiothorac Surg 14:S130-S133

32. Gurvitch R, Wood DA, Leipsic J et al (2010) Multislice computed tomography for prediction of optimal angiographic deployment projections during transcatheter aortic valve implantation. JACC Cardiovasc Interv 3:1157-1165

33. Kurra V, Kapadia SR, Tuzcu EM et al (2010) Pre-procedural imaging of aortic root orientation and dimensions: comparison between X-ray angiographic planar imaging and 3-dimensional multidetector row computed tomography. JACC Cardiovasc Interv 3:105-113

34. Tzikas A, Schultz C, Van Mieghem N et al (2010) Optimal projection estimation for transcatheter aortic valve implantation based on contrast-aortography: validation of a prototype software. Catheter Cardiovasc Interv 76:602-607

35. Falk V, Walther T, Schwammenthal E et al (2010) Transapical aortic valve implantation with a self-expanding anatomically oriented valve. Eur Heart $\mathbf{J}$ (in press)

36. Chow BJW, Abraham A, Wells GA et al (2009) Diagnostic accuracy and impact of computed tomographic coronary 
angiography on utilization of invasive coronary angiography. Circ Cardiovasc Imaging 2:16-23

37. Detaint D, Sundt TM, Nkomo VT et al (2006) Surgical correction of mitral regurgitation in the elderly: outcomes and recent improvements. Circulation 114:265-272

38. Mirabel M, Iung B, Baron G et al (2007) What are the characteristics of patients with severe, symptomatic, mitral regurgitation who are denied surgery? Eur Heart J 28: 1358-1365

39. Cleland JGF, Coletta AP, Buga L et al (2010) Clinical trials update from the American College of Cardiology meeting 2010: DOSE, ASPIRE, CONNECT, STICH, STOP-AF, CABANA, RACE II, EVEREST II, ACCORD, and NAVIGATOR. Eur J Heart Fail 12:623-629

40. Sack S, Kahlert P, Bilodeau L et al (2009) Percutaneous transvenous mitral annuloplasty. Circ Cardiovasc Interv 2:277-284

41. Siminiak T, Hoppe UC, Schofer J et al (2009) Effectiveness and safety of percutaneous coronary sinus-based mitral valve repair in patients with dilated cardiomyopathy (from the AMADEUS Trial). Am J Cardiol 104: 565-570

42. Schofer J, Siminiak T, Haude M et al (2009) Percutaneous mitral annuloplasty for functional mitral regurgitation: results of the CARILLON mitral annuloplasty device European Union study. Circulation 120:326-333

43. Grossi EA, Patel N, Woo YJ et al (2010) Outcomes of the RESTOR-MV trial (Randomized Evaluation of a Surgical Treatment for Off-pump Repair of the Mitral Valve). J Am Coll Cardiol 56:1984-1993

44. Feuchtner GM, Alkadhi H, Karlo C et al (2010) Cardiac CT angiography for the diagnosis of mitral valve prolapse: comparison with echocardiography. Radiology 254:374383

45. Delgado V, Tops LF, Schuijf JD et al (2009) Assessment of mitral valve anatomy and geometry with multislice computed tomography. JACC Cardiovasc Imaging 2:556-565

46. Gopal A, Shah A, Shareghi S et al (2011) The role of cardiovascular computed tomographic angiography for coronary sinus mitral annuloplasty. J Invasive Cardiol 22:67-73

47. Tops LF, Van de Veire NR, Schuijf JD et al (2007) Noninvasive evaluation of coronary sinus anatomy and its relation to the mitral valve annulus: implications for percutaneous mitral annuloplasty. Circulation 115:1426-1432

48. Choure AJ, Garcia MJ, Hesse B et al (2006) In vivo analysis of the anatomical relationship of coronary sinus to mitral annulus and left circumflex coronary artery using cardiac multidetector computed tomography: implications for percutaneous coronary sinus mitral annuloplasty. J Am Coll Cardiol 48:1938-1945

49. Chiribiri A, Kelle S, Kohler U et al (2008) Magnetic resonance cardiac vein imaging: relation to mitral valve annulus and left circumflex coronary artery. JACC Cardiovasc Imaging 1:729-738

50. Mishra YK, Mittal S, Jaguri P et al (2006) Coapsys mitral annuloplasty for chronic functional ischemic mitral regurgitation: 1-year results. Ann Thorac Surg 81:42-46

51. Palazzuoli A, Cademartiri F, Geleijnse ML et al (2010) Left ventricular remodelling and systolic function measurement with 64 multi-slice computed tomography versus second harmonic echocardiography in patients with coronary artery disease: a double blind study. Eur J Radiol 73:82-88 